\title{
Hard-thermal-loop resummed pressure of a degenerate quark-gluon plasma
}

\author{
Rudolf Baier ${ }^{1}$ and Krzysztof Redlich ${ }^{1,2}$ \\ ${ }^{1}$ Fakultät für Physik, Universität Bielefeld, \\ Postfach 100131 , D-33501 Bielefeld, Germany \\ ${ }^{2}$ Institute for Theoretical Physics, University of Wroctaw, PL-50204 Wroctaw, Poland
}

(March 21, 2018)

\begin{abstract}
We compute the pressure of a finite density quark-gluon plasma at zero temperature to leading order in hard-thermal-loop perturbation theory, which includes the fermionic excitations and Landau damping. The result is compared with the weak-coupling expansion for finite positive chemical potential $\mu$ through order $\alpha_{s}^{2}$ and with a quasiparticle model with a mass depending on $\mu$.
\end{abstract}

In this letter we extend the recent work by Andersen, Braaten and Strickland [1] by applying hard-thermal-loop (HTL) perturbation theory at the leading order [2] to a relativistic quark-gluon plasma at finite and positive chemical potential and at zero temperature. These investigations are motivated by the observation of the poor convergence properties of the perturbative expansion of thermodynamic functions describing the quark-gluon plasma at high temperature [3] and at high density [4,5]. However, efficient resummation schemes have been recently developed in the case of scalar field theory [6] and of gauge theories like QCD [1, /7], which improve considerably the convergence of the free energy at high temperature.

In the calculation of the high temperature behavior of the pressure of a gluon gas [1] the inverse HTL-resummed gluon propagator is important. In the case of a degenerate quark-gluon gas at $T=0$ and non-vanishing chemical potential $\mu$ the improved quark propagator plays the central role. The ideal gas behaviour at $T=0$ and $\mu \neq 0$ is determined by a quark loop

$$
P_{\text {ideal }}(\mu, T=0)=\lim _{T \rightarrow 0} \frac{T}{V} \operatorname{Tr} \log \not p=\frac{N_{c}}{12 \pi^{2}} \mu^{4},
$$

where we state the result for a one-flavor $\left(N_{f}=1\right)$ massless quark-gluon gas with $N_{c}=3$ colors. The fermionic Matsubara frequencies are given by $p_{0}=i(2 n+1) \pi T+\mu$. The $T=0$ limit is taken after the Matsubara sum is performed.

The HTL-pressure $P_{\mathrm{HTL}}$ receives contributions from three terms: $P_{\mathrm{HTL}}=P_{q}+P_{g}+\Delta P$, that is from the HTLresummed quark propagator, from the gluon propagator and from possible counterterms, which renormalize the pressure [1]. The quark-loop contribution $P_{q}$ is obtained by replacing the momentum $\not p$ in (11) by $\not p-\Sigma_{\mathrm{HTL}}$, where $\Sigma_{\text {HTL }}$ is the quark-selfenergy in the HTL approximation [8]. In the following we use the expressions and notations as given in [9], i.e.

$$
\not p-\Sigma_{\mathrm{HTL}}=A_{0} \gamma_{0}-A_{s} \vec{\gamma} \hat{p} \text { with } A_{0}=p_{0}-\frac{m_{f}^{2}}{p} Q_{0}\left(\frac{p_{0}}{p}\right) \text { and } A_{s}=p_{0}+\frac{m_{f}^{2}}{p}\left(1-\frac{p_{0}}{p} Q_{0}\left(\frac{p_{0}}{p}\right)\right),
$$

where $Q_{0}$ denotes the Legendre function. The quark thermal mass at $T=0$ and $\mu>0$ is given by

$$
m_{f}^{2}=\frac{N_{c}^{2}-1}{4 N_{c}} \frac{\alpha_{s}\left(\mu_{4}\right)}{\pi} \mu^{2}
$$

with the $\overline{M S}$ renormalization scale $\mu_{4}$. Therefore, for $N_{c}=3$ we have $m_{f}<\mu$. In terms of the functions $A_{0}, A_{s}$ the pressure is

$$
P_{q}=2 N_{c} y \log \left(A_{s}^{2}-A_{0}^{2}\right)
$$

where $\mathbb{E} \equiv T \sum_{n} \mu_{3}^{3-d} \int \frac{d^{d} p}{(2 \pi)^{d}}$ represents the sum-integral in $d$-dimension, $\mu_{3}$ denotes the $M S$ renormalization scale, necessary for the renormalization of the pressure using dimensional regularization for $d \neq 3$ dimensions [1].

In order to evaluate (4), it is useful to use the spectral representation of the quark-propagator. Having in mind the linear dependence of $A_{0}$ and $A_{s}$ on $m_{f}^{2}$ we first separate the ideal gas contribution by 


$$
P_{q}-P_{\text {ideal }}=\int_{0}^{m_{f}^{2}} d m^{2} \frac{\partial P_{q}}{\partial m^{2}}=2 N_{c} \int_{m_{f}^{2}}^{0} \frac{d m^{2}}{m^{2}} \bigvee\left\{\left(p_{0}-p\right) \Delta_{+}\left(p_{0}, p ; m^{2}\right)+\left(p_{0}+p\right) \Delta_{-}\left(p_{0}, p ; m^{2}\right)\right\} .
$$

For $\Delta_{ \pm}\left(p_{0}, p ; m^{2}\right)=\left(A_{0} \mp A_{s}\right)^{-1}$ the following dispersion relations hold

$$
\Delta_{ \pm}\left(p_{0}+i \eta, p ; m^{2}\right)=-\int_{-\infty}^{+\infty} \frac{d q_{0}}{2 \pi} \frac{\rho_{ \pm}\left(q_{0}, p ; m^{2}\right)}{q_{0}-p_{0}-i \eta}
$$

The sum rules for the HTL spectral function

$$
\int_{-\infty}^{+\infty} \frac{d p_{0}}{2 \pi}\left(p_{0} \mp p\right) \rho_{ \pm}\left(p_{0}, p ; m^{2}\right)=0
$$

turn out to be useful in our further discussion. Using (6) and (7) we find for the expression (5),

$$
P_{q}=P_{\text {ideal }}+2 N_{c} \int_{0}^{m_{f}^{2}} \frac{d m^{2}}{m^{2}} \int_{p} \int_{\mu}^{\infty} \frac{d p_{0}}{2 \pi}\left\{\left(p_{0}-p\right) \rho_{+}\left(p_{0}, p ; m^{2}\right)+\left(p_{0}+p\right) \rho_{-}\left(p_{0}, p ; m^{2}\right)\right\} .
$$

The derivation of (8) is based on the observation that the expression in the curly brackets of (5) is a meromorphic function in $p_{0}$, which decreases faster than $p_{0}^{-1}$ for $\left|p_{0}\right| \rightarrow \infty$, because of (7). This allows to perform the Matsubara frequency sum and to take the $T=0$ limit in the standard way [9].

The representation (8) covers the following contributions: (i) the fermion quasi-particle excitations for $p_{0}>p$, which are solutions of $\operatorname{Re} \Delta_{ \pm}^{-1}\left(p_{0}=\omega_{ \pm}, p\right)=0$. In (8) they contribute for $\omega_{ \pm}(p) \geq \mu$. (ii) the Landau damping contribution restricted by $\mu \leq p_{0} \leq p$.

In order to proceed we state the explicit expressions for the spectral functions,

$$
\begin{aligned}
\rho_{ \pm}\left(p_{0}, p ; m^{2}\right)= & 2 \pi\left[Z_{ \pm}(p) \delta\left(p_{0}-\omega_{ \pm}(p)\right)+Z_{\mp}(p) \delta\left(p_{0}+\omega_{\mp}(p)\right)\right]+\frac{\pi}{p} m^{2}(1 \mp x) \theta\left(1-x^{2}\right) \\
& \cdot\left[\left(p(1 \mp x) \pm \frac{m^{2}}{2 p}[(1 \mp x) L \pm 2]\right)^{2}+\frac{\pi m^{4}}{4 p^{2}}(1 \mp x)^{2}\right]^{-1}
\end{aligned}
$$

with $x=p_{0} / p, L=\log \left|\frac{x+1}{x-1}\right|$ and the residues at the quasi-particle poles $Z_{ \pm}(p)=\frac{\omega_{ \pm}^{2}(p)-p^{2}}{2 m^{2}}$. Next we consider the contributions (i) and (ii) separately and decompose

$$
P_{q}=P_{\text {ideal }}+P_{q, q u a s i}+P_{q, L d}+P_{q, s u b} .
$$

The quasi-particle contribution $P_{q, q u a s i}$ to the pressure $P_{q}$ is obtained as follows. Due to the $\delta$-functions in (9) the $p_{0}$-integration in (8) is straightforward. We also note the identities $d \omega_{ \pm}=\left(\omega_{ \pm} \mp p\right) Z_{ \pm}(p) \frac{d m^{2}}{m^{2}}$, which allow to perform the $m^{2}$-integration. The quark $(+)$ mode contribution has an ultraviolet divergence for $d=3$ dimensions. Following the procedure of [i] this divergence is isolated by subtracting expressions from the integrands to obtain finite results for $d=3$, and evaluating the subtracted integrals in $d=3-2 \epsilon,(\epsilon>0)$ dimensions. The subtracted terms take into account that $\omega_{+} \rightarrow p+\frac{m_{f}^{2}}{p}-\frac{m_{f}^{4}}{2 p^{3}} \log \frac{2 p^{2}}{m_{f}^{2}}$, for $p>>m_{f}$ [8]. The quark $(-)$ mode contribution is ultraviolet finite, therefore it does not require subtracted terms. The finite quasi-particle contribution for $d=3$ reads

$$
\begin{aligned}
P_{q, q u a s i}=2 N_{c} \int \frac{d^{3} p}{(2 \pi)^{3}} & \left\{\left(\omega_{+}(p)-\mu\right) \theta\left(\omega_{+}-\mu\right) \theta(\mu-p)+\left(\omega_{-}(p)-\mu\right) \theta\left(\omega_{-}-\mu\right) \theta(\mu-p)\right. \\
+ & \left(\omega_{-}(p)-p\right) \theta(p-\mu)-\left(\omega_{+}(p)-p\right) \theta(\mu-p)+\left[\omega_{+}(p)\right. \\
& \left.\left.-\sqrt{p^{2}+2 m_{f}^{2}}+\frac{m_{f}^{4}}{2\left(p^{2}+2 m_{f}^{2}\right)^{3 / 2}}\left(\log \frac{2\left(p^{2}+2 m_{f}^{2}\right)}{m_{f}^{2}}-1\right)\right]\right\} .
\end{aligned}
$$

The Landau damping term $P_{q, L d}$ is treated in an analogous way. The dependence of $\rho_{ \pm}$on $m^{2}$ allows to perform the $m^{2}$-integration in (8) explicitly. The ultraviolet divergence, which is also present in this contribution, is treated as described above. The finite term for $d=3$ becomes

$$
\begin{aligned}
P_{q, L d}=-4 N_{c} \int \frac{d^{3} p}{(2 \pi)^{3}} \theta(p-\mu) \int_{\mu}^{p} & \frac{d p_{0}}{2 \pi}\left[\Phi_{+}\left(p_{0}, p ; m_{f}^{2}\right)-\Phi_{-}\left(p_{0}, p ; m_{f}^{2}\right)\right. \\
& \left.+\frac{\pi m_{f}^{4} p_{0}}{p^{3}} \frac{1}{\left(p^{2}-p_{0}^{2}+2 m_{f}^{2}\right)}+\frac{\pi m_{f}^{4}}{2 p^{4}} \log \left(\frac{p+p_{0}}{p-p_{0}}\right)\right],
\end{aligned}
$$


where the angles $\Phi_{ \pm}$are given by

$$
\Phi_{ \pm}\left(p_{0}, p ; m_{f}^{2}\right)=\arctan \frac{\pi(1 \mp x) m_{f}^{2}}{2 p\left[p(1 \mp x)+\frac{m_{f}^{2}}{2 p}(2 \pm(1 \mp x) L)\right]} .
$$

The terms in (12) which are subtracted in order to make it finite are constructed by observing that $\Phi_{+}-\Phi_{-} \rightarrow$ $-\frac{\pi m_{f}^{4}}{2 p^{4}}\left[2 x /\left(1-x^{2}+2 m_{f}^{2} / p^{2}\right)+L\right]$ for $p>>m_{f}$.

Finally, we discuss the term $P_{q, s u b}$, which contains the renormalized subtracted contributions evaluated in the limit $\epsilon \rightarrow 0$. We note that the quasi-particle and Landau damping terms in (8) have poles proportional to $1 / \epsilon^{2}$ and to $1 / \epsilon$, which however cancel when adding these two contributions. Therefore no counter term is required:

$$
\Delta P_{q}=0
$$

The remaining finite term reads

$$
P_{q, \text { sub }}=N_{c} \frac{m_{f}^{4}}{4 \pi^{2}}\left[-\frac{1}{2}\left(\log \frac{m_{f}^{2}}{2 \mu^{2}}\right)^{2}-4 \log 2+\frac{5}{2}-\int_{0}^{-\log \frac{2 m_{f}^{2}}{\mu^{2}}} \frac{t d t}{e^{t}-1}\right] .
$$

We observe from (15) that $P_{q}$ includes terms of $O\left(m_{f}^{4} \hat{=} \alpha_{s}^{2}\right)$. Consequently we have to consider contributions of $O\left(m_{g}^{4} \hat{=} \alpha_{s}^{2}\right)$ arising from the HTL-resummed gluon loop. In leading order the result is already given in 返 when discussing the $T=0$ limit of the pressure, namely by

$$
P_{g}=-\left(N_{c}^{2}-1\right) \frac{9}{64 \pi^{2}} m_{g}^{4}\left(\log \frac{m_{g}}{\overline{\mu_{3}}}-0.332837\right),
$$

where the $\overline{M S}$ scale ${\overline{\mu_{3}}}^{2}=4 \pi e^{-\gamma} \mu_{3}^{2}$ is introduced. However, here we have to replace the thermal gluon mass, which is $m_{g}^{2}=\frac{4 \pi \alpha_{s}}{3} T^{2}$ in a hot gluon gas, by $m_{g}^{2}=\frac{2}{3} \frac{\alpha_{s}}{\pi} \mu^{2}$ [10] for the case of degenerate quark-gluon gas. We note that $m_{g}$ coincides with $m_{f}$. In the following we fix the renormalization scale $\overline{\mu_{3}}$ by $\overline{\mu_{3}}=0.717 m_{f}$, such that $P_{g}=0$, and only the quark-loop contributes to $P_{\text {HTL }}$ (besides the proper counter term for the gluon-loop).

For later comparisons with the weak-coupling expansion we expand $P_{q}$ in powers of $m_{f} / \mu<<1$, and we find

$$
\frac{P_{q}}{P_{\text {ideal }}} \simeq 1-2 N_{c} \frac{m_{f}^{2}}{\mu^{2}}+O\left(\frac{m_{f}^{4}}{\mu^{4}}\right)=1-4 \frac{\alpha_{s}}{\pi}+O\left(\alpha_{s}^{2}\right) .
$$

We note that the leading correction of $O\left(m_{f}^{2} / \mu^{2}\right)$ is exclusively due to the quark $(+)$ mode contained in $P_{q, q u a s i}$ of (11).

The pressure of a degenerate quark-gluon plasma is calculated through order $\alpha_{s}^{2}$ in the weak-coupling expansion [4.

$$
P_{Q C D}=P_{\text {ideal }}\left[1-2 \frac{\alpha_{s}\left(\mu_{4}\right)}{\pi}-\left(9.267+\log \left(\frac{\alpha_{s}\left(\mu_{4}\right)}{\pi}\right)-\frac{31}{3} \log \frac{\mu}{\mu_{4}}\right)\left(\frac{\alpha_{s}\left(\mu_{4}\right)}{\pi}\right)^{2}\right]
$$

with $\frac{\alpha_{s}\left(\mu_{4}\right)}{\pi}=\frac{12}{31 L}\left(1-\frac{804}{961} \frac{\log \bar{L}}{L}\right)$ for $N_{c}=3$ and $\bar{L}=\log \left(\mu_{4}^{2} / \Lambda_{\overline{M S}}^{2}\right)$.

Although $P_{Q C D}$ in (18) depends on $\mu_{4}$ beginning at order $\alpha_{s}^{3}$, the dependence on $\mu_{4}$ turns out to be rather strong. For $\mu / \Lambda_{\overline{M S}}=2, P_{Q C D} / P_{\text {ideal }}$ varies from 0.68 to 0.82 under the variation from $\mu_{4}=2 \mu$ to $\mu_{4}=8 \mu$. With increasing $\mu / \Lambda \overline{M L S}$ the variation obviously decreases, and consequently the difference between the $O\left(\alpha_{s}\right)$ and $O\left(\alpha_{s}^{2}\right)$ contributions in $(18)$ is diminished. This is shown in Fig.1 for the choice $\mu_{4}=4 \mu$.

When comparing the $O\left(\alpha_{s}\right)$ correction term of $P_{Q C D}$ in (18) with the one of the HTL-resummed pressure $P_{\text {HTL }}$ at $T=0$ (17) we note a difference in magnitude by a factor 2 . As in the case of the gluon gas at high $T$ [1] HTL resummation overestimates the $O\left(\alpha_{s}\right)$ term. In order to correct for this difference, we add a correction term to $P_{\mathrm{HTL}}$, namely $P_{\text {corr }}=2 \frac{\alpha_{s}}{\pi} P_{\text {ideal }}$. We define the pressure

$$
P \equiv P_{\mathrm{HTL}}+P_{\text {corr }}
$$

and attribute $P_{\text {corr }}$ to quasi-particle interactions [1]. Presently, however, we are only able to approximate the corresponding series for $P_{\text {corr }}$ by its leading $O\left(\alpha_{s}\right)$ contribution. 


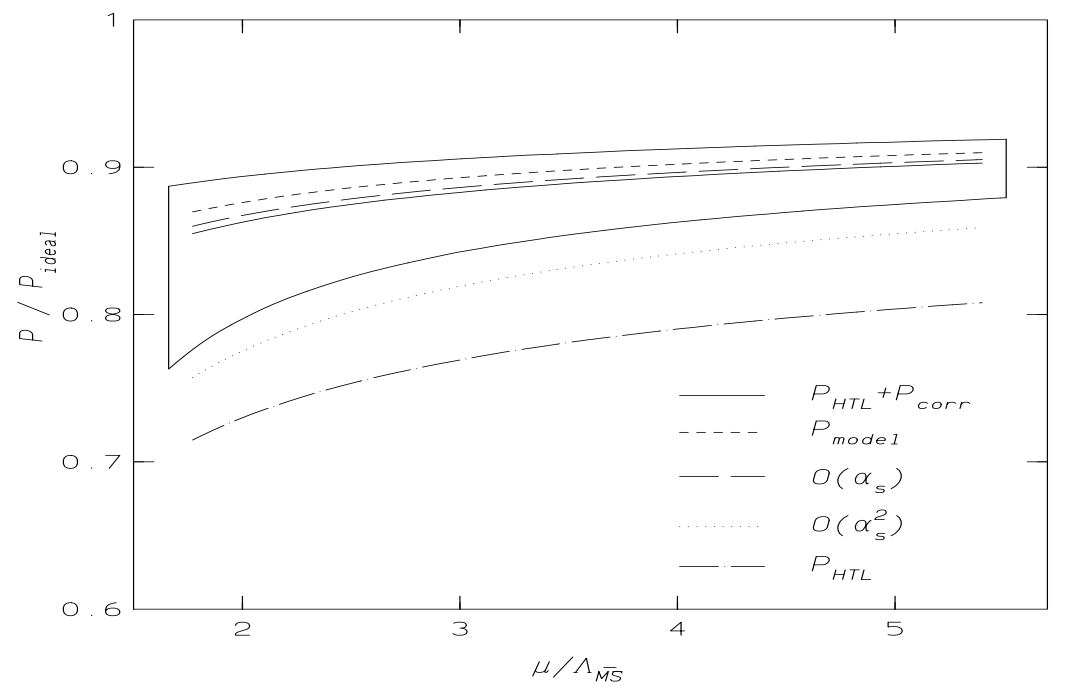

FIG. 1. The pressure for a degenerate quark-gluon plasma normalized to the ideal gas result as a function of $\mu / \Lambda \overline{M S}$. The $P_{H T L}+P_{\text {corr }}$ pressure is shown for $\mu_{4}=4 \mu$ and as a band that corresponds to varying $\mu_{4}$ from $\mu_{4}=2 \mu$ to $\mu_{4}=8 \mu$ (solid line). The weak-coupling expansions through order $O\left(\alpha_{s}\left(\mu_{4}\right)\right)$ and $O\left(\alpha_{s}^{2}\left(\mu_{4}\right)\right)$ are shown by long-dashed and dotted lines correspondingly; the HTL pressure $P_{H T L}$ is indicated by the dashed-dotted line, and the pressure $P_{\text {model }}$ for non-interacting massive, $m=m_{f}\left(\mu_{4}\right)$, fermionic quasi-particles is shown as short-dashed line. All are calculated for $\mu_{4}=4 \mu$.

In Fig. 1 we show $P_{\mathrm{HTL}}$ as a function of $\mu / \Lambda_{\overline{M S}}$ evaluated for $\mu_{4}=4 \mu$, indicating the overestimate of the $O\left(\alpha_{s}\right)$ correction. Numerically the main contribution to $P_{\mathrm{HTL}}$ is due to the quark mode $\omega_{+}(p)$ in (11), which is arising from the momentum interval $\mu \geq p>0$. The result for $P$ is plotted in Fig. 1 for $\mu_{4}=4 \mu$ and as a band which corresponds to the variation of the scale $\mu_{4}$ by $2 \mu \leq \mu_{4} \leq 8 \mu$. We observe that the $O\left(\alpha_{s}^{2}\right)$ corrections of the weak-coupling expansion (18) lead to a much bigger deviation from the ideal gas behaviour than the HTL-resummed $P$, which follows more closely the $O\left(\alpha_{s}\right)$ behaviour of (18) in the considered region of $\mu / \Lambda_{\overline{M S}}$.

We can not compare at present our result for the pressure at $T=0, \mu>0$, with lattice results, as it could be done at $\mu=0$ and $T>0$. Instead we evaluate $P$ for non-interacting massive, $m \neq 0$, fermionic quasi-particles $\left(N_{f}=1, N_{c}=3\right)$ with finite chemical potential. The corresponding pressure reads [1]

$$
P_{\text {model }}=\frac{m^{4}}{4 \pi^{2}}\left[\frac{\mu}{m}\left(\frac{\mu^{2}}{m^{2}}-1\right)^{\frac{1}{2}}\left(\frac{\mu^{2}}{m^{2}}-\frac{5}{2}\right)+\frac{3}{2} \log \left[\frac{\mu}{m}+\sqrt{\frac{\mu^{2}}{m^{2}}-1}\right],\right.
$$

with $\mu>m$. Choosing $m=m_{f}$ of (3) we realize that through $O\left(\alpha_{s}\right) P_{\text {model }}$ coincides with $P_{Q C D}(18)$ as well as with $P(19)$. This is clearly seen in Fig.1 where $P_{\text {model }}$ is plotted for $\mu_{4}=4 \mu$. Indeed the quasi-particle approximation 12 turns out to be an excellent effective description of the degenerate quark-gluon plasma at $T=0$, when the quasiparticle mass $m$ is identified with the quark thermal mass $m_{f}$ of the HTL approach. This agreement is also shown in Fig.2, where we summarize different approximations for the pressure discussed in this letter, which we normalize to the ideal gas for $T=0, m=0$ and positive $\mu$. For the choice $\mu_{4}=4 \mu$ we plot in Fig.2 the ratio $P / P_{\text {ideal }}$ as a function of the coupling $\alpha_{s}\left(\mu_{4}\right)$ in the region $0 \leq \alpha_{s} \leq 0.4$ for $P_{\mathrm{HTL}}, P=P_{\mathrm{HTL}}+P_{\text {corr }}, P_{Q C D}$ through $O\left(\alpha_{s}\right)$ and $O\left(\alpha_{s}^{2}\right)$, and for $P_{\text {model }}$.

Pisarski and Rischke in a recent paper [13] conjecture that perturbation theory at $T=0, \mu>0$ may work better than for $T>0$, i.e. they estimate its breakdown when the coupling becomes as large as $\alpha_{s} \sim 2.4$. From Fig.2 one may estimate a "critical" $\alpha_{s} \sim 0.4$, i.e. where the $\alpha_{s}^{2}$ term becomes as big as the $\alpha_{s}$ term of $P_{Q C D}$. At $T>0$ a corresponding estimate gives $\alpha_{s} \sim 0.1-0.2$.

Only after the inclusion of, at least, the next-to-leading order correction in the HTL-perturbation scheme one could judge if the prediction for the pressure of a degenerate quark-gluon plasma becomes considerably more stable, especially when compared to the weak-coupling expansion (18). Then it would be extremely interesting [14] to compare with numerical lattice results for the $T=0$ finite density plasma which also, however, still have to be worked out. 


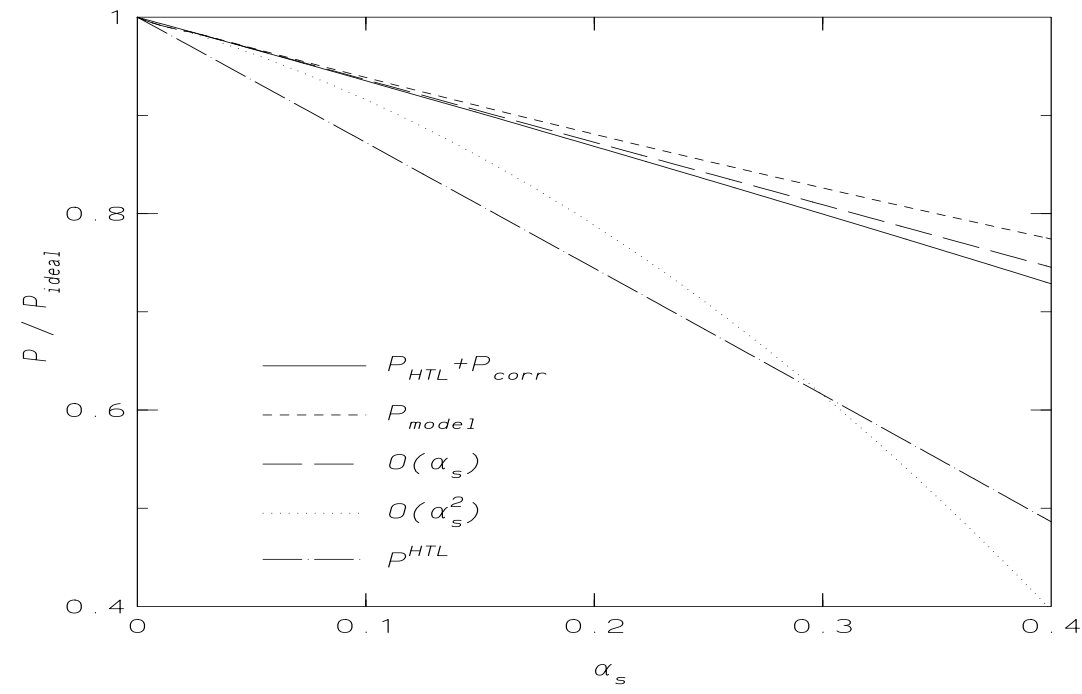

FIG. 2. The pressure for a degenerate quark-gluon plasma normalized to the ideal gas result as a function of $\alpha_{s}\left(\mu_{4}\right)$. The contributions to the pressure correspond to those of Fig.1, consistently calculated with $\mu_{4}=4 \mu$.

After having completed this letter we have found a relevant new paper by Andersen, Braaten, and Strickland 15 where the quark contribution to the free energy of a hot $(T \neq 0)$ but baryon free $(\mu=0)$ quark-gluon plasma is calculated to leading order in HTL-perturbation theory. In another recent paper [16] the calculation of the baryon density in a self-consistent approximation in the sense of [7, 17] is presented as a function of $\mu / \Lambda_{\overline{M S}}$.

This work is supported in part by Deutsche Forschungsgemeinschaft (DFG project KA 1198/4-1). We thank H. Satz for interesting discussions and A. Rebhan for useful comments. We especially wish to thank J. O. Andersen for pointing out to us a mistake in Eqs. (12) and (15) of the early version of this paper. One of us (K.R.) also acknowledges stimulating discussions with F. Karsch and D. E. Miller.

[1] J. O. Andersen, E. Braaten, and M. Strickland, Phys. Rev. Lett. 83, 2139 (1999); hep-ph/9905337.

[2] R. D. Pisarski, Phys. Rev. Lett. 63, 1129 (1989); E. Braaten, and R. D. Pisarski, Nucl. Phys. B337, 569 (1990); J. Frenkel, and J. C. Taylor, Nucl. Phys. B334, 199 (1990).

[3] P. Arnold, and C. Zhai, Phys. Rev. D51, 1906 (1995); E. Braaten, and A. Nieto, Phys. Rev. D53, 3421 (1996); for a review see e.g. A. Smilga, Phys. Rept. 271, 1 (1997).

[4] B. A. Freedman, and L. D. McLerran, Phys. Rev. D16, 1169 (1978); T. Toimela, Int. J. Theoret. Phys. 24, 901 (1985).

[5] J. I. Kapusta, "Finite-Temperature Field Theory", Cambridge University Press, Cambridge, England, 1989.

[6] B. Kastening, Phys. Rev. D56, 8107 (1997); T. Hatsuda, Phys. Rev. D56, 8111 (1997); F. Karsch, A. Patkos, and P. Petreczky, Phys. Lett. B401, 69 (1997); I. T. Drummond, R. R. Horgan, P. V. Landshoff, and A. Rebhan, Nucl. Phys. B524, 579 (1998); A. Rebhan, hep-ph/9808480.

[7] J. P. Blaizot, E. Iancu, and A. Rebhan, Phys. Rev. Lett. 83, 2906 (1999).

[8] V. V. Klimov, Sov. Phys. JETP 55, 199 (1982); H. A. Weldon, Phys. Rev. D26, 2789 (1982) and D40, 2410 (1989) ; R. D. Pisarski, Nucl. Phys. A498, 423c (1989).

[9] M. Le Bellac, "Thermal Field Theory", Cambridge University Press, Cambridge, England, 1996.

[10] T. Toimela, Phys. Lett. B176, 463 (1986).

[11] J. Cleymans, R. Gavai, and E. Suhonen, Phys. Rept. 130, 217 (1986).

[12] J. Engels, J. Fingberg, K. Redlich, H. Satz, and M. Weber, Z. Phys. C42, 341 (1989); V. Goloviznin, and H. Satz, Z. Phys. C57, 671 (1993); A. Peshier, B. Kämpfer, O. P. Pavlenko, and G. Soff, Phys. Lett. B337, 235 (1994), and Phys. Rev. D54, 2399 (1996).

[13] R. D. Pisarski and D. H. Rischke, nucl-th/9907041, R. D. Pisarski (private communication). 
[14] F. Wilczek, hep-ph/9907340.

[15] J. O. Andersen, E. Braaten, and M. Strickland, hep-ph/9908323.

[16] J. P. Blaizot, E. Iancu, and A. Rebhan, hep-ph/9910309.

[17] G. Baym, Phys. Rev. 127, 1391 (1962); B. Vanderheyden and G. Baym, J. Stat. Phys. 93, 843 (1998). 